\title{
An orthogonal technique for empirical mode decomposition in Hilbert- Huang transform
}

\author{
Menglin Lou ${ }^{1, a}$ and Tianli Huang ${ }^{2}$ \\ ${ }^{1}$ Tongji University, School of Civil Engineering, Shanghai, China \\ ${ }^{2}$ Central South University, School of Civil Engineering, Changsha, China
}

\begin{abstract}
First, it is indicated that the intrinsic mode functions (IMF) obtained by the empirical mode decomposition (EMD) are not orthogonal. Then an orthogonal technique based on Gram-Schmidt method is proposed to obtain the complete orthogonal intrinsic mode functions. Three ways of the orthogonal processes are suggested and compared in the paper. Finally, the suggested method is validated through the decomposition of a typical time history. The numerical results show that the orthogonal IMF can be obtained easily by the technique proposed in the paper.
\end{abstract}

\section{Intrinsic mode functions}

Hilbert-Huang transform is applied widely in the signal analysis process as well as in Earthquake Engineering ${ }^{[1-3]}$. After the empirical mode decomposition (EMD), the signal $X(t)$ can be expressed as

$$
X(t)=\sum_{j=1}^{n} c_{j}(t)+r_{n}(t)
$$

where $c_{j}(t)$ is the $j^{\text {th }}$ intrinsic mode function (IMF), $r_{n}(t)$ is the residual function.

The integral orthogonal index IOT and the orthogonal index $I O_{j k}$ between the $j^{\text {th }}$ IMF and the $k^{\text {th }}$ IMF are designated respectively for checking the orthogonality of the IMFs.

$$
\begin{gathered}
I O T=\sum_{j=1}^{n+1} \sum_{\substack{k=1 \\
k \neq j}}^{n+1} \int_{0}^{T} c_{j}(t) c_{k}(t) d t / \int_{0}^{T} X^{2}(t) d t \\
I O_{j k}=\frac{\int_{0}^{T} c_{j}(t) c_{k}(t) d t}{\int_{0}^{T} c_{j}^{2}(t) d t+\int_{0}^{T} c_{k}^{2}(t) d t}
\end{gathered}
$$

If all of IMFs are orthogonal mutually, the values of $I O T$ and $I O_{j k}$ are zero. However, the orthogonality of the IMFs can not be verified by mathematical technique. The following simple example shows the IMFs are not orthogonal. The signal $X(t)$ is assumed as the sum of three sinusoidal waves

$$
X(t)=\sum_{j=1}^{3} x_{j}(t)=\sum_{j=1}^{3} \sin \left(2 \pi f_{j} t\right)
$$

where $f_{1}=1 \mathrm{~Hz}, f_{2}=5 \mathrm{~Hz}, f_{3}=10 \mathrm{~Hz}$. Its time history curve with 5 seconds is shown in Figure 1. Obviously, three sinusoidal waves are orthogonal, the theoretical values of $I O T$ and $I O_{j k}$ are zero. The numerical values of
$I O T$ and $I O_{j k}$ are very small if they are calculated by computer, such as $I O T=2.5545 \mathrm{e}-016$ and

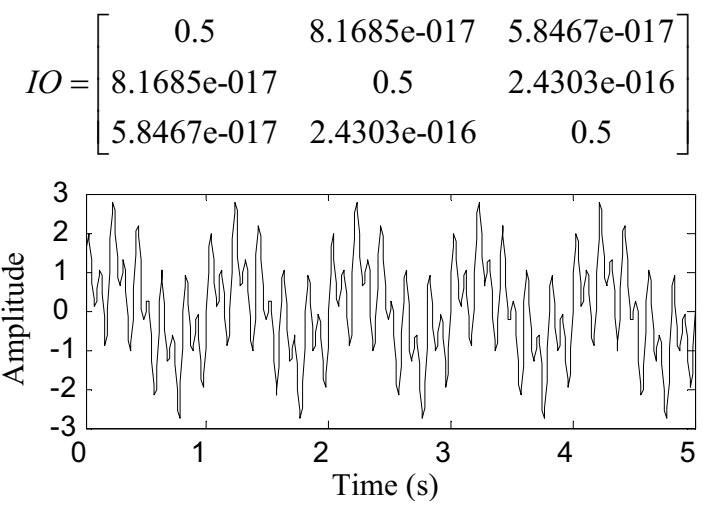

Figure 1. Time history curve of a simple signal



Figure 2. The original IMF components (EMD)

\footnotetext{
a Corresponding author: 1ml@tongji.edu.cn, 13701910214@163.com
} 
The signal $X(t)$ can be decomposed as five IFMs and one residual function when applying the EMD, shown in Figure 2. These five IMFs are defined as original IMFs.

The orthogonal index IOT $=0.06633$ and $I O_{j k}$ are shown in Table 1. Comparing with the orthogonal level of three sinusoidal components, the orthogonal level of the original IMFs drops a lot.

Table 1. The values of the orthogonal index $I O_{j k}$ (original IMFs)

\begin{tabular}{|c|c|c|c|c|c|}
\hline $\begin{array}{c}\text { Order } \\
j, k\end{array}$ & 1 & 2 & 3 & 4 & 5 \\
\hline 1 & 0.5 & $1.1 \mathrm{E}-01$ & $3.9 \mathrm{E}-03$ & $8.9 \mathrm{E}-05$ & $3.2 \mathrm{E}-05$ \\
\hline 2 & $1.1 \mathrm{E}-01$ & 0.5 & $4.9 \mathrm{E}-02$ & $2.1 \mathrm{E}-04$ & $8.6 \mathrm{E}-05$ \\
\hline 3 & $3.9 \mathrm{E}-03$ & $4.9 \mathrm{E}-02$ & 0.5 & $1.5 \mathrm{E}-03$ & $5.2 \mathrm{E}-04$ \\
\hline 4 & $8.9 \mathrm{E}-05$ & $2.1 \mathrm{E}-04$ & $1.5 \mathrm{E}-03$ & 0.5 & $3.0 \mathrm{E}-01$ \\
\hline 5 & $3.2 \mathrm{E}-05$ & $8.6 \mathrm{E}-05$ & $5.2 \mathrm{E}-04$ & $3.0 \mathrm{E}-01$ & 0.5 \\
\hline
\end{tabular}

\section{Orthogonal technique for original IMFs}

Gram-Schmidt method is applied usually for obtaining the orthogonal eigenvectors or Ritz vectors in structural dynamics $^{[4]}$. This method is used here to achieve the orthogonal IMFs from the original IMFs. The process to obtain the orthogonal IMFs is defined as improved EMD (IEMD). There are three different ways to get the orthogonal IMFs $c_{j}^{*}(t)$ from the original IMFs $c_{j}(t)$. The signal $X(t)$ is expressed by the orthogonal IMFs as

$$
X(t)=\sum_{j=1}^{n} c_{j}^{*}(t)+r_{n}(t)
$$

\subsection{Forward sequence orthogonalization}

The original IMFs obtained from Eq.(1) are sequenced from high frequency to low frequency. In Gram-Schmidt orthogonalization, the sequence order is not changed. The computing steps of the orthogonalization are introduced as follows ${ }^{[5]}$.

(1) The first basic orthogonal function is taken as the first original IMF, that is

$$
d_{1}(t)=c_{1}(t)
$$

(2) The second basic orthogonal function is formed from the second original IMF $c_{2}(t)$, but orthogonal to the first basic function $d_{1}(t)$.

$$
d_{2}(t)=c_{2}(t)-\beta_{21} d_{1}(t)
$$

in which $\beta_{21}$ is defined as the orthogonal factor between $c_{2}(t)$ and $d_{1}(t)$ and obtained from

$$
\begin{gathered}
\int_{0}^{T} d_{1}(t) d_{2}(t) d t=\int_{0}^{T} c_{2}(t) d_{1}(t) d t-\beta_{21} \int_{0}^{T} d_{1}^{2}(t) d t=0 \\
\beta_{21}=\frac{\int_{0}^{T} c_{2}(t) d_{1}(t) d t}{\int_{0}^{T} d_{1}^{2}(t) d t}
\end{gathered}
$$

(3) Recurrent formula for solving high order basic orthogonal functions,

$$
d_{j+1}(t)=c_{j+1}(t)-\sum_{i=1}^{j} \beta_{j+1, i} d_{i}(t)
$$

Utilizing the orthogonality of the first $j$ basic orthogonal functions and equation

$$
\begin{gathered}
\int_{0}^{T} d_{j+1}(t) d_{k}(t) d t=\int_{0}^{T} c_{j+1}(t) d_{k}(t) d t \\
-\sum_{i=1}^{j} \beta_{j+1, i} \int_{0}^{T} d_{k}(t) d_{i}(t) d t=0
\end{gathered}
$$

when $\mathrm{k}=\mathrm{i}$, we can obtain

$$
\beta_{j+1, i}=\frac{\int_{0}^{T} c_{j+1}(t) d_{i}(t) d t}{\int_{0}^{T} d_{i}^{2}(t) d t}
$$

(4) Substitute Eqs.(6), (7) and (9) into Eq. (1), the signal $X(t)$ can be described as

$$
\begin{gathered}
X(t)=c_{1}^{*}(t)+c_{2}^{*}(t)+c_{3}^{*}(t)+\cdots+c_{j}^{*}(t)+\cdots c_{n-1}^{*}(t)+c_{n}^{*}(t)+r_{n}(t) \\
=\sum_{j=1}^{n} c_{j}^{*}(t)+r_{n}(t)=\sum_{j=1}^{n} a_{j} d_{j}(t)+r_{n}(t)
\end{gathered}
$$

where $a_{j}=\sum_{i=j}^{n} \beta_{i, j}(j=1,2, \cdots, n), \beta_{i, j}=1(i=j) \cdot c_{j}^{*}(t)$ is the $j^{\text {th }}$ orthogonal IMF that are sequenced from high frequency to low frequency.

\subsection{Backward sequence orthogonalization}

In Gram-Schmidt orthogonalization, the sequence order is changed reversely. It means that the computing steps are modified as follows.

(1) The first basic orthogonal function is taken as the last original IMF, that is

$$
d_{1}(t)=c_{n}(t)
$$

(2) The second basic orthogonal function is formed from the last second original IMF $c_{n-1}(t)$, but orthogonal to the first basic function $d_{1}(t)$.

$$
d_{2}(t)=c_{n-1}(t)-\beta_{21} d_{1}(t)
$$

in which $\beta_{21}$ is defined as the orthogonal factor between $c_{n-1}(t)$ and $d_{1}(t)$ and obtained from

$$
\beta_{21}=\frac{\int_{0}^{T} c_{n-1}(t) d_{1}(t) d t}{\int_{0}^{T} d_{1}^{2}(t) d t}
$$

(3) Recurrent formula for solving high order basic orthogonal functions,

$$
\begin{gathered}
d_{j+1}(t)=c_{n-j}(t)-\sum_{i=1}^{j} \beta_{j+1, i} d_{i}(t) \\
\beta_{j+1, i}=\frac{\int_{0}^{T} c_{n-j}(t) d_{i}(t) d t}{\int_{0}^{T} d_{i}^{2}(t) d t}
\end{gathered}
$$

(4) Substitute Eqs.(13), (14) and (16) into Eq. (1), the signal $X(t)$ can also be described by Eq. (12). However, the difference is that the orthogonal IMFs $c_{j}^{*}(t)$ are sequenced from low frequency to high frequency. 
Obviously, if the work of rearranging reversely the sequence order of the original IMFs is finished at first, the orthogonal computations can be completed by Eqs. (6)-(12).

\subsection{Arbitrary sequence orthogonalization}

In Gram-Schmidt orthogonalization, the sequence order is changed arbitrarily. It means that any one of these $n$ orthogonal IMFs can be selected as the first basic orthogonal function and the sequence order of these $n$ original IMFs can be rearranged if necessary. After the sequence order rearrangement, the computing steps of the Gram-Schmidt orthogonalization are the same introduced in section 2.1. The difference is that the orthogonal IMFs $c_{j}^{*}(t)$ are not sequenced from low frequency to high frequency or from high frequency to low frequency.

\section{Numerical example}

The signal $X(t)$ shown in Eq.(4) is continuously used as the example. The comprisons of the numerical results obtained from different orthogonal methods are shown as follows. In arbitrary sequence method, the $3^{\text {th }}$ original IMF is selected as the first basic orthogonal IMF. The following is the $2^{\text {nd }}$, the $1^{\text {st }}$, the $4^{\text {th }}$ and $5^{\text {th }} \mathrm{IMF}$.

\subsection{Orthogonal IMF components}

The orthogonal IMFs are shown in Figures (3)-(5).

\subsection{Orthogonal index}

The values of integral orthogonal index IOT are 0.000504 (IEMD-1), 0.000613 (IEMD-2) and 0.000452 (IEMD-3) respectively. The values of the orthogonal index $I O_{j k}$ are listed in Tables 2-4.

It is obvious that the orthogonal level of the IMFs has been greatly improved when the orthogonal technique is applied to the original IMFs.

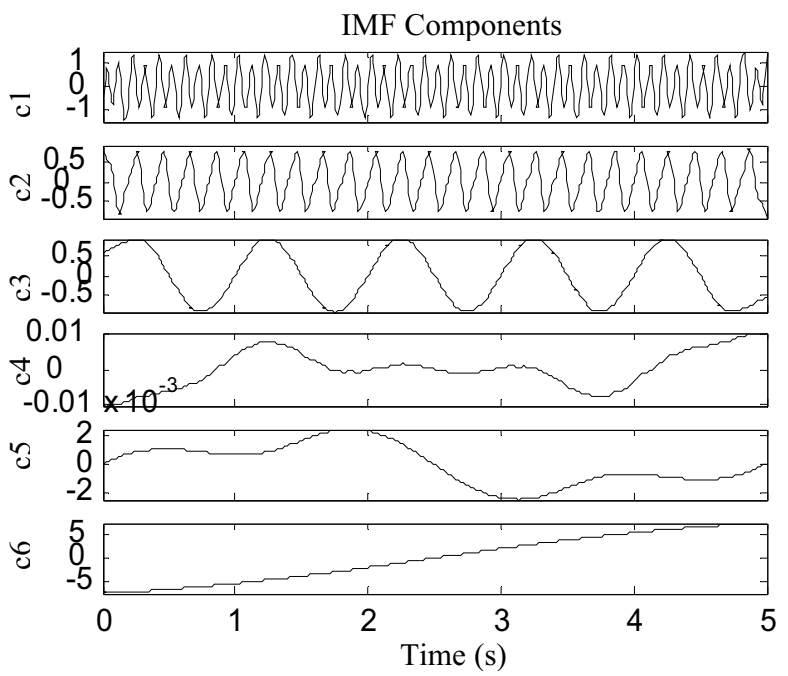

Figure 3. The orthogonal IMF components (IEMD-1)

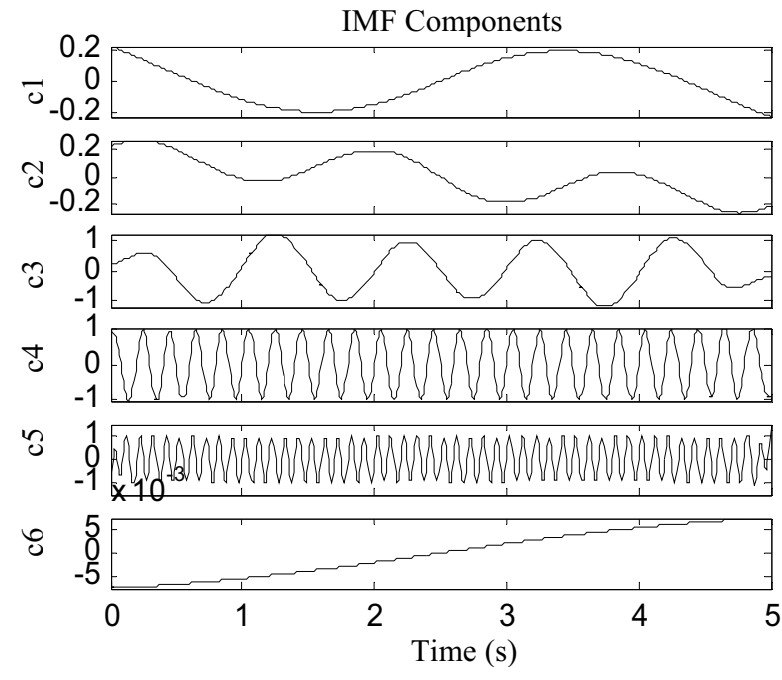

Figure 4. The orthogonal IMF components (IEMD-2)

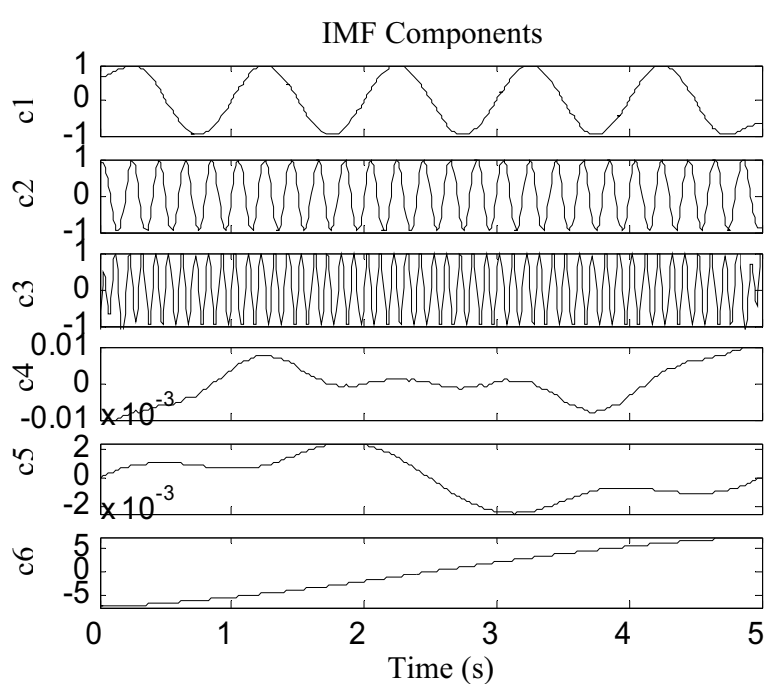

Figure 5. The orthogonal IMF components (IEMD-3)

Table 2. The values of the orthogonal index $I O_{j k}$ (IEMD -1)

\begin{tabular}{|c|c|c|c|c|c|}
\hline $\begin{array}{c}\text { Order } \\
j, k\end{array}$ & 1 & 2 & 3 & 4 & 5 \\
\hline 1 & 0.5 & $3.2 \mathrm{E}-17$ & $2.7 \mathrm{E}-18$ & $4.4 \mathrm{E}-20$ & $8.5 \mathrm{E}-21$ \\
\hline 2 & $3.2 \mathrm{E}-17$ & 0.5 & $8.2 \mathrm{E}-18$ & $3.1 \mathrm{E}-19$ & $5.2 \mathrm{E}-20$ \\
\hline 3 & $2.7 \mathrm{E}-18$ & $8.2 \mathrm{E}-18$ & 0.5 & $1.3 \mathrm{E}-18$ & $1.2 \mathrm{E}-19$ \\
\hline 4 & $4.4 \mathrm{E}-20$ & $3.1 \mathrm{E}-19$ & $1.3 \mathrm{E}-18$ & 0.5 & $3.2 \mathrm{E}-16$ \\
\hline 5 & $8.5 \mathrm{E}-21$ & $5.2 \mathrm{E}-20$ & $1.2 \mathrm{E}-19$ & $3.2 \mathrm{E}-16$ & 0.5 \\
\hline
\end{tabular}

Table 3. The values of the orthogonal index $I O_{j k}$ (IEMD -2)

\begin{tabular}{|c|c|c|c|c|c|}
\hline $\begin{array}{c}\text { Order } \\
j, k\end{array}$ & 1 & 2 & 3 & 4 & 5 \\
\hline 1 & 0.5 & $2.3 \mathrm{E}-18$ & $1.8 \mathrm{E}-17$ & $2.9 \mathrm{E}-18$ & $2.1 \mathrm{E}-18$ \\
\hline 2 & $2.3 \mathrm{E}-18$ & 0.5 & $3.6 \mathrm{E}-17$ & $2.5 \mathrm{E}-18$ & $1.1 \mathrm{E}-18$ \\
\hline 3 & $1.8 \mathrm{E}-17$ & $3.6 \mathrm{E}-17$ & 0.5 & $8.4 \mathrm{E}-18$ & $9.0 \mathrm{E}-19$ \\
\hline 4 & $2.9 \mathrm{E}-18$ & $2.5 \mathrm{E}-18$ & $8.4 \mathrm{E}-18$ & 0.5 & $7.2 \mathrm{E}-17$ \\
\hline 5 & $2.1 \mathrm{E}-18$ & $1.1 \mathrm{E}-18$ & $9.0 \mathrm{E}-19$ & $7.2 \mathrm{E}-17$ & 0.5 \\
\hline
\end{tabular}


Table 4. The values of the orthogonal index $I O_{j k}$ (IEMD -3)

\begin{tabular}{|c|c|c|c|c|c|}
\hline $\begin{array}{c}\text { Order } \\
j, k\end{array}$ & 1 & 2 & 3 & 4 & 5 \\
\hline 1 & 0.5 & $4.5 \mathrm{E}-18$ & $4.8 \mathrm{E}-18$ & $1.8 \mathrm{E}-18$ & $8.0 \mathrm{E}-20$ \\
\hline 2 & $4.5 \mathrm{E}-18$ & 0.5 & $8.6 \mathrm{E}-17$ & $1.6 \mathrm{E}-19$ & $3.8 \mathrm{E}-20$ \\
\hline 3 & $4.8 \mathrm{E}-18$ & $8.6 \mathrm{E}-17$ & 0.5 & $3.3 \mathrm{E}-19$ & $3.6 \mathrm{E}-20$ \\
\hline 4 & $1.8 \mathrm{E}-18$ & $1.6 \mathrm{E}-19$ & $3.3 \mathrm{E}-19$ & 0.5 & $1.6 \mathrm{E}-16$ \\
\hline 5 & $8.0 \mathrm{E}-20$ & $3.8 \mathrm{E}-20$ & $3.6 \mathrm{E}-20$ & $1.6 \mathrm{E}-16$ & 0.5 \\
\hline
\end{tabular}

\subsection{Importance of orthogonality}

The orthogonality of the IMFs has an important effect on its applications. It is illustrated by a simple example. If the signal $X(t)$ is a seismic wave, its vibrating energy can be computed by

$$
E_{x}=\int_{0}^{T} X^{2}(t) d t
$$

The energy of each component in the seismic wave can be expressed

$$
E_{j}=\int_{0}^{T} c_{j}^{2}(t) d t \quad(j=1, \cdots, n+1)
$$

If the wave components are orthogonal, there are

$$
\begin{gathered}
E_{t o t}=\sum_{j=1}^{n+1} E_{j}=E_{x} \\
E_{j k}=\int_{0}^{T} c_{j}(t) c_{k}(t) d t=0 \quad(j, k=1, \cdots, n+1 ; j \neq k)
\end{gathered}
$$

Now we compute the energy of the signal composed by three sinusoidal waves shown in Eq.(4). Total energy
$E_{\text {tot }}=750$ and the energy between the sinusoidal components is listed in the following matrix. Its total energy is equal to the sum of three component energy. The numerical results satisfiy Eqs. (20) and (21) as the sinusoidal functions are orthogonal.

$$
E=\left[\begin{array}{ccc}
250 & -4.08 \mathrm{e}-014 & 2.92 \mathrm{e}-014 \\
-4.08 \mathrm{e}-014 & 250 & 1.22 \mathrm{e}-013 \\
2.92 \mathrm{e}-014 & 1.22 \mathrm{e}-013 & 250
\end{array}\right]
$$

When the signal $X(t)$ is decomposed by EMD and improved EMD respectively, the values of its $E_{t o t}$ and $E_{j}$ are listed in Table 5. It can be seen from numerical results in Table 5 that tatal energy of the signal $X(t)$ in EMD method is underestimated as the IMFs are not orthogonal that causes energy leakage between the components. Three IEMD methods improve the situation and ensure high calculation accuracy for total energy of the signal. However, the calculation accuracy of component energy is different for three IEMD methods. IEMD-3 is the best to get good results. This is the problem to be payed attention to.

\section{Conclusion}

The improved empirical mode decomposition has been

\begin{tabular}{|c|c|c|c|c|c|c|c|c|c|}
\hline Method & $\mathrm{E}_{\mathrm{x}}$ & $\mathrm{E}_{1}$ & $\mathrm{E}_{2}$ & $\mathrm{E}_{3}$ & $\mathrm{E}_{4}$ & $\mathrm{E}_{5}$ & $\mathrm{E}_{6}$ & $\mathrm{E}_{\text {tot }}$ & Error(\%) \\
\hline EMD & \multirow{4}{*}{750} & 265.84 & 136.65 & 259.76 & 0.0076 & 0.002 & 0.0119 & 662.28 & -11.7 \\
\hline IEMD-1 & & 358.39 & 133.58 & 258.65 & 0.0134 & 0.0009 & 0.0119 & 750.64 & 0.09 \\
\hline IEMD-2 & & 8.8587 & 11.52 & 238.12 & 241.14 & 250.99 & 0.0119 & 750.64 & 0.09 \\
\hline IEMD-3 & & 258.49 & 240.93 & 251.19 & 0.0134 & 0.0009 & 0.0119 & 750.64 & 0.09 \\
\hline
\end{tabular}
developed to obtain the orthogonal intrinsic mode function. The numerical results show that the method has good calculation accuracy.

Table 5. The values of total energy and component energy

\section{References}

1. N.E. Huang, S.R. Long, Proc. R. Soc. Lond. A, 454, 903-995 (1998)

2. Y.L. Xu, J. Chen, J. of Engineering Mechanics, 130, 1279-1288 (2004)
3. R. Zhang, S. Ma, E. Safak, S. Hartzell, J. of Engineering Mechanics, 129, 861-875 (2003)

4. R.W. Clough, J. Penzien, Dynamics of structures ( McGraw-Hill, Inc, New York, 1993)

5. M. Lou, T. Huang, J. of Tongji Universty, 35, 293298 (2007) (in Chinese) 